\title{
Nutritional status in the elderly: misbeliefs, misconceptions and the real world
}

\author{
Mitja Lainscak · Cristiana Vitale
}

Published online: 29 November 2016

(C) Springer-Verlag Wien 2016

Nutritional status and body habitus are closely related throughout the lifespan and generally follow a standard trajectory. Both can be modified, primarily due to the life style of an individual, and fluctuations in body weight are frequently observed in the modern era. These mostly occur in cycles of weight gain, followed by voluntary weight loss because of aesthetic reasons. In the elderly and patients with chronic disease, however, weight loss generally is not a good sign; in fact, the contrary is true and the so-called obesity paradox with reverse epidemiology is an established term [1-4].

Although weight loss is common and frequently reported in clinical medicine, there is a considerable diversity in terminology as used in clinical practice and even among researchers to describe the patient body habitus and nutritional status [5]. It is therefore not surprising that many misbeliefs and misconceptions are present in the field. Seen from the evolutionary perspective, a default presumption is that true beliefs are adaptive and misbeliefs maladaptive [6]. The question therefore is, why people make exceptions to true beliefs and why misbeliefs are present and even so frequent. Generally, there are two types of misbelief: they can be due to a breakdown in the normal functioning of the belief formation system

\footnotetext{
M. Lainscak, MD, PhD, FESC, FHFA ( $₫)$

Department of Cardiology and Department of Research and

Education, General Hospital Celje, Oblakova 5, 3000 Celje, Slovenia

mitja.lainscak@guest.arnes.si

M. Lainscak, MD, PhD, FESC, FHFA

Faculty of Medicine, University of Ljubljana, Ljubljana, Slovenia

C. Vitale, MD, PhD

Department of Medical Sciences, IRCCS San Raffaele Pisana, Rome, Italy
}

(e.g. delusions) or they can arise due to incomplete or inaccurate information (but with a normal course of the belief formation system). The latter can definitely be observed in the literature and in clinical practice $[1,5,7]$. This can in part be due to lack of gold standards for many definitions in the fields of nutrition, body composition and body habitus as criteria are often descriptive and arbitrary. Terms such as "anorexia", "malnutrition/undernutrition", "sarcopenia", "cachexia" and even "frailty" are commonly used as synonyms, even by the experts in the field [5]. One can argue that all are mostly associated with weight change but this is not necessarily true, e.g. in sarcopenia; some of them look beyond nutrition itself as they also consider patient performance (frailty, sarcopenia) [2, 8]. Evidently, there is some overlap between them and some per definition coexist (malnutrition and cachexia) in the same patient. There are pathophysiological grounds for this as several mechanisms are shared and perpetuate each other [7, 9-11]. It is therefore of utmost importance that uniform terminology is used for research and clinical practice and that comparable methods are used to assess the patient's nutritional status and performance (Fig. 1). In this issue of the Journal, several papers have touched on these issues and they elegantly demonstrate that significant inconsistency persists in the field, both in general terms and in geriatrics [12-14]. This is why the recent publications of the European Society of Parenteral and Enteral Nutrition (ESPEN) are so important to minimize or even abolish the heterogeneity in the field [15, 16]. Once such a consensus is reached, research activity and yield across the field should increase, as should the investigator and industry-driven research. From the clinical point of view, it is also important to evaluate nutritional risks so patient risks can be stratified better and managed if evidence-based therapy exists 


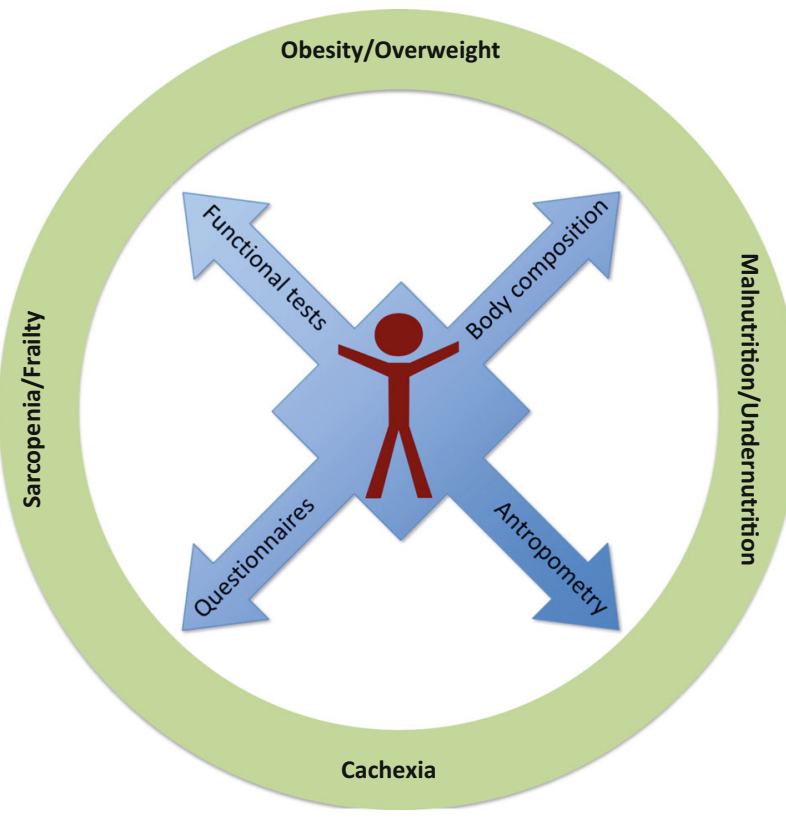

Fig. 1 Nutritional evaluation and nutritional profiles

[17-23]. Some nutritional problems can easily be treated and those are mostly linked to malnutrition/ undernutrition and micronutrient abnormalities. As nutritional issues commonly go hand in hand with chronic disease, certain caveats regarding the pharmacotherapy exist. Drug pharmacokinetics depend on body composition and organ function that can be altered due to nutritional disorders with or without chronic disease thus caution or referral to a clinical pharmacist should be considered [24, 25].

In pursuit of better patient assessment (Fig. 1), existing evidence from large epidemiological databases should be examined in detail. The nutritionDay, a multinational cross-sectional snapshot survey, definitely holds a wealth of information that is relevant and can identify processes that can easily be addressed [26, 27]. Generally, a golden hour for nutritional assessment is during hospitalization when patients are available for several days and are more susceptible to potential interventions. The real world is certainly not meeting this challenge as such services could be delivered in less than half of the participating hospitals in the nutritionDay survey. Nutritional status assessment and risk stratification are evidently insufficient but area necessary step to appreciate the magnitude of the problem. The next and maybe even greater challenge is the sustainable system to deliver appropriate management if evidence is available. The latter is still largely lacking thus sufficiently powered and well-designed interventional studies using nutritional support are crucial to close the loop [28].

Conflict of interest M. Lainscak and C. Vitale declare that they have no competing interests.

\section{References}

1. Lainscak M, Filippatos GS, Gheorghiade M, Fonarow GC, Anker SD. Cachexia: common, deadly, with an urgent need for precise definition and new therapies. Am J Cardiol. 2008;101:8E-10E.

2. von Haehling S, Anker SD. Prevalence, incidence and clinical impact of cachexia: facts and numbers-update 2014. J Cachexia Sarcopenia Muscle. 2014;5:261-3.

3. Farkas J, von HaehlingS, Kalantar-ZadehK, MorleyJE, Anker SD, LainscakM. Cachexia as a major publichealth problem: frequent, costly, and deadly. JCachexia Sarcopenia Muscle. 2013;4:173-8.

4. Lainscak M, von Haehling S, Doehner W, Anker SD. The obesity paradox in chronic disease: facts and numbers. JCachexia Sarcopenia Muscle. 2012;3:1-4.

5. Ter Beek L, Vanhauwaert E, Slinde F, et al. Unsatisfactory knowledge and use of terminology regarding malnutrition, starvation, cachexia and sarcopenia among dietitians. Clin Nutr. 2016;35:1450-6.

6. McKay RT, Dennett DC. The evolution of misbelief. Behav Brain Sci. 2009;32:493-510.

7. Ebner N, Springer J, Kalantar-Zadeh K, et al. Mechanism and novel therapeutic approaches to wasting in chronic disease. Maturitas. 2013;75:199-206.

8. MorleyJE, AnkerSD, von Haehling S. Prevalence, incidence, and clinical impact of sarcopenia: facts, numbers, and epidemiology-update 2014. J Cachexia Sarcopenia Muscle. 2014;5:253-9.

9. Anker SD, Morley JE. Cachexia: a nutritional syndrome? JCachexia Sarcopenia Muscle. 2015;6:269-71.

10. Alchin DR. Sarcopenia: describing rather than defining a condition. JCachexia Sarcopenia Muscle. 2014;5:265-8.

11. Molfino A, Papa A, Gasperini-Zacco ML, et al. Left ventricular mass correlates with lean body mass in patients with disease-associated wasting. J Cachexia Sarcopenia Muscle. 2014;5:251-2.

12. Morley JE. Frailty and sarcopenia in elderly. Wien Klin Wochenschr. 2016; doi:10.1007/s00508-016-1087-5.

13. Saitoh M, Ebner N, Emami A, et al. Nutritional status and its effects on muscle wasting in patients with chronic heart failure: Insights from the Studies Investigating Comorbidities Aggravating Heart Failure (SICA-HF). Wien Klin Wochenschr. 2016; doi:10.1007/s00508-016-1112-8.

14. Springer J, Anker SD. Publication trends in cachexia and sarcopenia in elderly heart failure patients. Wien Klin Wochenschr. 2016; doi:10.1007/s00508-016-1126-2.

15. Cederholm T, Barazzoni R, Austin P, et al. ESPEN guidelines on definitions and terminology of clinical nutrition. Clin Nutr. 2016; doi:10.1016/j.clnu.2016.09.004.

16. Cederholm T, Bosaeus I, Barazzoni R, et al. Diagnostic criteria for malnutrition-An ESPEN consensus statement. Clin Nutr. 2015;34:335-40.

17. Gu W, Zhang G, Sun L, et al. Nutritional screening is strongly associated with overall survival in patients treated with targeted agents for metastatic renal cell carcinoma. JCachexia Sarcopenia Muscle. 2015;6:222-30.

18. Henwood TR, Keogh JW, Reid N, Jordan W, Senior HE. Assessing sarcopenic prevalence and risk factors in residential aged care: methodology and feasibility. J Cachexia Sarcopenia Muscle. 2014;5:229-36.

19. Villani AM, Miller MD, Cameron ID, Kurrle S, Whitehead C, Crotty M. Development and relative validity of a new field instrument for detection of geriatric cachexia: preliminary analysis in hip fracture patients. J Cachexia Sarcopenia Muscle. 2013;4:209-16. 
20. Fearon K, Argiles JM, Baracos VE, et al. Request for regulatory guidance for cancer cachexia intervention trials. J Cachexia Sarcopenia Muscle. 2015;6:272-4.

21. MorleyJE, Cao L. Rapid screening for sarcopenia. JCachexia Sarcopenia Muscle. 2015;6:312-4.

22. Lainscak M, von Haehling S, Doehner W, et al. Body mass index and prognosis in patients hospitalized with acute exacerbation of chronic obstructive pulmonary disease. JCachexia Sarcopenia Muscle. 2011;2:81-6.

23. Benedik B, Farkas J, Kosnik M, Kadivec S, Lainscak M. Mini nutritional assessment, body composition, and hospitalisations in patients with chronic obstructive pulmonary disease. Respir Med. 2011;105(Suppl 1):S38-S43.

24. Cvan Trobec K, Kerec Kos M, Trontelj J, et al. Influence of cancer cachexia on drug liver metabolism and renal elimination in rats. J Cachexia Sarcopenia Muscle. 2015;6:45-52.
25. Trobec K, Kerec Kos M, von Haehling S, Springer J, Anker SD, Lainscak M. Pharmacokinetics of drugs in cachectic patients: a systematic review. PLOS ONE. 2013;8:e79603.

26. Schindler K, Themessl-Huber M, Hiesmayr M, et al. To eat or not to eat? Indicators for reduced food intake in 91,245 patients hospitalized on nutritionDays 2006-2014 in 56 countries worldwide: a descriptive analysis. Am J Clin Nutr. 2016;104:1393-402.

27. Lainscak M, Farkas J, Frantal S, et al. Self-rated health, nutritional intake and mortality in adult hospitalized patients. Eur JClin Invest. 2014;44:813-24.

28. Deutz NE, Matheson EM, Matarese LE, et al. Readmission and mortality in malnourished, older, hospitalized adults treated with a specialized oral nutritional supplement: A randomized clinical trial. Clin Nutr. 2016;35:18-26. 